\title{
Liver transplant at Dow University of health sciences (DUHS) Karachi, Pakistan
}

Keywords: chronic liver, acute, morbidity, mortality, liver disease, poor tolerance, cardiopulmonary status, frailty, morbid obesity, active infection, active substance abuse, poor psychosocial support system, laboratory, wait list, crucial numbers, candidacy

Abbrevations: LT, liver transplant; DUHS, dow university of health sciences karachi; NILGID, national institute of liver \& GI diseases

\section{Mini review}

The evolution of liver transplant has come a long way since the 1980 's when it got internationally recognized as a viable treatment for end-stage liver disease. In 1963, Dr. Thomas Starzl and his team performed the first human liver transplant in the United States. However it was not until the 1980's that the survival rates improved enough to consider liver transplantation a clinical treatment for acute and chronic liver failure, and not an experimental procedure. Roy Calne, a professor of surgery at Cambridge introduced cyclosporine in 1980s and observed excellent patient outcomes post liver transplantation.

Liver transplantation is a viable treatment option for end-stage liver disease and acute liver failure. The most commonly used technique is Orthotopic transplantation in which the native liver is removed and replaced by the donor organ in the same anatomic location. Liver transplantation is now performed worldwide with more than 100 centers in the U.S alone.

With improvements in surgical technique, patient selection, organ procurement as well as post-transplant care including availability of newer classes of immunosuppressive agents, not only post-transplant survival increased significantly but also the number of total transplants performed and patients on waiting list also multiplied. As a result of increasing success rates for improving survival, liver transplant as a treatment got more widely applied to increasing number of liver ailments that were previously considered a relative or even an absolute contraindication. Currently any patient with acute or chronic liver failure producing significant morbidity and mortality risk is a potential candidate for LT. Now practically, the few reasons to not consider LT as a viable treatment option for a patient with worsening liver disease is either poor overall medical condition that predicts poor tolerance of high risk surgical procedure, specially poor cardiopulmonary status, frailty, morbid obesity, active infection, active substance abuse, poor psychosocial support system, incurable extra hepatic malignancy and brain death.

The most common indication for liver transplantation is viral hepatitis related liver failure, mainly chronic or end-stage. Hepatitis C is the most frequent prevalent culprit. Reliable data on epidemiology of liver disease and its etiologies is from the western hemisphere. Pakistan is particularly lagging behind in collecting basic data on incidence and prevalence of diseases as well as associated mortality rates. This area needs urgent attention as without these crucial

\author{
Volume 3 Issue I - 2017
}

\author{
Khawar S Jamali \\ Pro-Vice Chancellor, Dow University of Health Sciences \\ (DUHS), Pakistan
}

Correspondence: Khawar S Jamali, Dow University of Health Sciences (DUHS) Karachi, Pakistan, Email pro-vc@duhs.edu.pk

Received: July 31, 2016 | Published: March 09, 2017

numbers, the scope of the problem cannot be defined well and therefore problem-solving efforts will remain inferential.

Limited data indicates that prevalence of Hepatitis B carrier state and Hepatitis B past exposure is about $10 \%$ and $33 \%$ respectively amongst Pakistanis. These figures are unfortunately from the 1980's and later data is at least not searchable on PubMed or Medline even if it exists. Local health bodies agree that actual figures in present time are expected to be drastically higher as risk factors for acquiring hepatitis B or C still prevail and may have worsened. Therefore it can be extrapolated that there is a significant percentage of patient population in need of evaluation for liver transplantation and presently only a limited few have the resources to travel outside Pakistan and afford liver transplant in other countries. The remaining large majority eventually succumbs to acute or chronic liver disease when medical therapy becomes refractory. With fewer restrictions on candidacy, the need to make liver transplantation accessible is even greater.

In Pakistan May 2016, Dow University of Health Sciences Karachi (DUHS) became the first public sector university to offer liver transplant facilities. Our team under supervision has done three consecutive living-donor liver transplants. Post-surgical recovery for all 3 recipients and their donors has been uneventful and they are recovering as per expected parameters. Our overseas team mentor who supervised these procedures has successfully performed nearly 2000 liver transplants and has helped the DUHS team along with his other team members. We currently have three patients on the wait list, expecting transplantation in the following month.

Additionally DUHS has got its own state of the art laboratory, with latest automated testing equipment. It has one of the best imaging departments with latest CT and MRI scanners.

The other facility catering to the liver and GI related ailments was National Institute of Liver \& GI Diseases (NILGID). It is functioning since Sept 2010 and daily more than 100 patients are seen in the OPD. Seriously ill patients are admitted to the hospital with availability of intensive care unit. There are two endoscopy suites performing regular and specialized diagnostic and therapeutic procedures. This institute also provides ultrasound guided liver \& GI interventions. Radio frequency ablation is available to treat liver and metastatic malignancies. Emergency services for upper or lower gastrointestinal cases are also available round the clock. ${ }^{1-5}$ 
Moreover DUHS is doing its duty to increase public awareness of liver diseases as well as living and cadaveric organ donation. With an expectant large waiting list, development of the services depends on the availability of trained professionals including surgeons and support staff for which places like our unit, which although new and novice, has been built on strong foundations and matches international standards. We now need sincere international collaboration and support from world renowned liver transplantation centers for growth and improvement so we can provide even better patient care.

\section{Acknowledgements}

None.

\section{Conflict of interest}

Author declares that there is no conflict of interest.

\section{References}

1. Starzl Thomas E, Anthony J Demetris, David Van Thiel. Medical progress: liver transplantation. $N$ Engl J Med. 1989;321(15):1014-1022.

2. Seaberg Eric C, Belle SH, Beringer KC, et al. Liver transplantation in the United States from 1987-1998: updated results from the Pitt-UNOS Liver Transplant Registry. Clin transpl. 1998;17-37.

3. Lake JR. Changing indications for liver transplantation. Gastroenterol Clin North Am. 1993;22(2):213-229.

4. Tong CY, Khan R, Beeching NJ, et al. The occurrence of hepatitis B and C viruses in Pakistani patients with chronic liver disease and hepatocellular carcinoma. Epidemiol infect. 1996;117(2):327-332.

5. Malik IA, Luqman M, Ahmad A. A clinico- pathological study of viral hepatitis. Pakistan J Med Res. 1987;26:4-11. 